\title{
Magneto-rotational and Thermal Evolution of Magnetars with Crustal Magnetic Fields
}

\section{U. Geppert}

Astrophysikalisches Institut Potsdam, An der Sternwarte 16, 14482

Potsdam, GERMANY, e-mail: urme@aip.de

D. Page

Instituto de Astronomía, UNAM, 04510 Mexico D.F., MEXICO, e-mail: page@astroscu.unam.mx

M. Colpi

Dipartimento di Fisica, Universitá degli Studi di Milano Bicocca, Via Emanueli 15,20133 Milano,Italy,colpi@uni.mi.astro.it

\section{T. Zannias}

Instituto de Física y Matemáticas, Universidad Michoacana SNH, Morelia, Mich.58040, MEXICO,e-mail: zannias@ginette.ifm.umich.mx

\section{Introduction}

The interpretation of Soft-Gamma-Repeaters (SGRs) and Anomalous X-Ray Pulsars (AXPs) as Magnetars (Thompson \& Duncan 1996) raises again the issue of the generation of the ultra-strong magnetic fields (MFs) in neutron stars (NSs) and the related question of where these fields are anchored: in the core, penetrating the whole star, or confined to the crust. Recently, Heyl \& Kulkarni (1998) considered the magneto-thermal evolution of magnetars with a core field. Since the assumption of a crustal field is at least not in disagreement with the observations of isolated pulsars (Urpin \& Konenkov 1997) and of NSs in binary systems (Urpin, Geppert \& Konenkov 1998, Urpin, Konenkov \& Geppert 1998), here we would like to address the question whether the observations of SGRs and AXPs can be interpreted as magnetars having a crustal MF. Given the strength of the $\mathrm{MF}$ in magnetars we take into account, in an approximate manner, the strongly non-linear Hall effect on its decay. We intend to provide a contribution to an unified picture of NS MF evolution based on the crustal field hypothesis.

\section{Model}

We consider three qualitatively different equations of state (EOS), a stiff one from Pandharipande et al. (1976), a medium one from Wiringa et al. (1988) and a soft one from Pandharipande (1971) which includes the effect of hyperons. We consider for all EOSs the possibility of both slow neutrino emission from the modified Urca processes and fast neutrino emission by the direct Urca processes; 
all standard neutrino emission processes in the crust are also properly taken into account (see, e.g., Page, 1998). The thermal evolution is essentially affected by the different neutrino emissivities, however, in the case of magnetars Joule heating and the effect of the strongly magnetized envelope modify the thermal history considerably compared to that of standard NSs. The Joule heating rate is determined by $j^{2} / \sigma_{\text {eff }}$, where $j$ is the current density and $\sigma_{\text {eff }}$ the electrical conductivity (see below). Since the calculation is performed under spherical symmetry this value is averaged over spheres of constant radius. Ultra-intense MFs affect strongly the transport of heat in the upper layers of the star, the envelope, resulting in a higher flux than in the case of weaker fields (Heyl \& Hernquist 1998). This implies that young magnetars will be naturally hotter than standard young NSs even if their interior followed the same thermal path. We, moreover, assume here that the envelope is made of iron: an envelope containing light elements would lead to an even higher flux. We solve the general relativistic equations of heat conservation and transport in the whole star by using a Henyey type code developed specially for NS cooling studies (see Page 1998 and references therein). We consider the evolution of a purely poloidal dipolar MF, maintained by currents in the crust of the NS. It is governed by

$$
\frac{1}{c} \frac{\partial \vec{B}}{\partial t}+\vec{\nabla} \wedge\left[\frac{c}{4 \pi \sigma_{\text {eff }}} \vec{\nabla} \wedge\left(e^{\Phi} \vec{B}\right)\right]=0 \quad \text { with } \quad \sigma_{\text {eff }}=\sigma_{\text {eff }}(T, \rho, \vec{B}, \ldots)
$$

The Hall effect will not directly decay the MF, but will redistribute magnetic energy into field modes with smaller length scales (the HALL CASCADE, Goldreich \& Reisenegger, 1992) which may decay faster, thereby accelerating the decay of the large scale dipolar field too. Since we present here only results of one-dimensional calculations we cannot modelize the process of the generation of small-scale field modes and their interaction with the dipolar mode. Instead we mimic the effect of the Hall drift upon the field decay of its dipolar mode by using an effective electrical conductivity

$$
\sigma_{\mathrm{eff}}=\sigma_{\mathrm{ohm}} / \sqrt{1+\left(\omega_{B} \tau\right)^{2}}
$$

where $\omega_{B}$ is the gyrofrequency of electrons and $\tau$ is their relaxation time. This simple expression for $\sigma_{\text {eff }}$ reproduces the two limiting cases of a weak field (i.e., $\sigma=\sigma_{\mathrm{ohm}}$ ) and of a strong field (i.e., $\sigma=\sigma_{\mathrm{ohm}} / \omega_{B} \tau$ ). The $\sigma_{\mathrm{ohm}}$ takes into account the different collision mechanisms of the electrons that can appear in the crust (see Urpin et al., 1998). The metric function $\Phi(r)$ is related by Einstein's equations to the density and pressure distribution within the crust and describes the general relativistic effects which decelerate the field decay (Geppert, Page \& Zannias, 1999). We solve the induction equation simultaneously with the equations of the thermal evolution by use of a Crank-Nicholson scheme. The spin-history is calculated adopting the simple dipolar radiation losses equation.

\section{Results}

The evolution of the rotational period $P$ and its derivative $\dot{P}$, of the surface MF $B_{s}$ and of the luminosity $L$ for the model which most promisingly coincides with observations is represented in figures 1 and 2 . The evolution of magnetars differs qualitatively from that of standard NSs. While the latter ones reach 


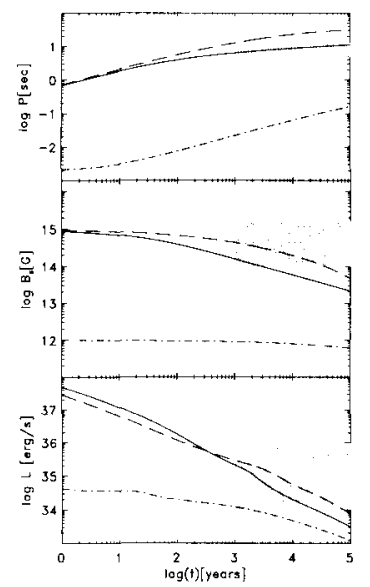

Figure 1. Evolution of $P, B_{s}$, and $L$ for a model with a stiff EOS, standard cooling scenario and an initial field of $B_{s 0}=10^{15} \mathrm{G}$ which penctrates initially the crust up to densities $\rho_{0}=10^{13} \mathrm{~g} \mathrm{~cm}^{-3}$ (full lines) and $10^{14} \mathrm{~g} \mathrm{~cm}^{-3}$ (dashed lines). For comparison we show by the dot dashed lines the evolution for the same model $B_{s 0}=10^{12} \mathrm{G}$ and $\rho_{0}=10^{14} \mathrm{~g} \mathrm{~cm}^{-3}$. The shaded areas indicate the regions of typical values observed for magnetars.

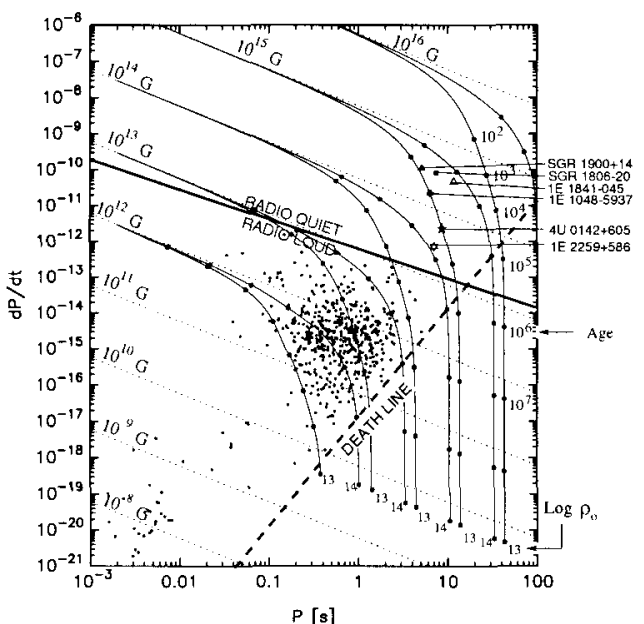

Figure 2. Evolutionary tracks for the most promising model: stiff EOS and standard cooling. The diagram contains the positions of all pulsars (dots) with $\dot{P}$ measurements from the Princeton Pulsar Cata$\log$, the 'pulsar dead line' as well as 'radio quiescence line' above which the generation of the magnetospheric pair plasma is quenched by photon splitting. The positions of SGRs and AXPs are shown. The ages of the models are indicated by the fat dots. $\log \left(\rho_{0}\right)$ is indicated on each curve. Notice that a larger $\rho_{0}$ implies slower decay and thus larger periods are reached. 
isothermality after a few $100 \mathrm{yrs}$, the decay of an ultra-strong MF in the crust causes an enormous heat release which can be appreciated comparing the evolution of the luminosity of a standard NS with that of magnetars (lower panel of figure 1). The delicate interplay between Joule heating, heat transport and neutrino cooling results in significant temperature gradients in the whole crust for at least $10^{4}$ years. To describe the observations the field decay must be, on the one hand, slow enough to lead to large rotational periods and keep magnetic energy stored in the crust for a long time but, on the other hand, fast enough to produce an intense Joule heating and to cluster the final rotational periods around 8 s (see Colpi, Geppert \& Page 1999). Those delicate requirements can be accomplished by a crustal MF.

\section{Discussion}

The assumption of a crustal field is not in contradiction with observations of AXPs, provided the EOS is stiff enough and a standard cooling scenario applies. Furthermore, the initial MF strength should lie in a rather narrow range around $10^{15} \mathrm{G}$. This result may inspire speculations about a possible bimodal distribution of initial MFs of NSs. The majority of NSs is born with canonical fields of $10^{12 \pm 1} \mathrm{G}$ while, probably due to another field generation mechanism, the magnetars are born with $10^{15 \pm 0.5} \mathrm{G}$. The crustal field hypothesis predicts naturally the existence of a critical saturation field of a few $10^{15} \mathrm{G}$. Above this threshold the magnetic energy is preferentially conveyed into mechanical energy by fracturing the crystallized crust (Thompson \& Duncan 1996). The rather stable spin-down of AXPs indicates that their field is just below this saturation value.

Concluding we can state that the crustal field hypothesis, although simply describing the Hall drift and its consequences, provides a framework for explaining the thermal evolution, luminosity and period clustering of magnetars.

\section{References}

Colpi, M., Geppert, U. \& Page D. 1999, ApJ, submitted

Geppert, U., Page D. \& Zannias, T. 1999, Phys.Rev.D, in press

Goldreich, P. \& Reisenegger, A. 1992, ApJ, 395, 250

Heyl, J. S.\& Hernquist, L. 1998, MNRAS, 300, 599

Heyl, J. S.\& Kulkarni, S. R. 1998, ApJ, 506, L61

Pandharipande, V.R. 1971, Nucl. Phys., A 178, 123

Pandharipande, V.R., Pines, D. \& Smith, R.A. 1976, ApJ, 208, 550

Page, D. 1998, in Neutron Stars and Pulsars, eds N. Shibazaki, N. Kawai, S. Shibata, \& T. Kifune, (Universal Academy Press: Tokyo), p. 183.

Thompson, C. \& Duncan, R. C. 1996, ApJ, 473, 322

Urpin, V.A. \& Konenkov, D. Yu. 1997, MNRAS, 292, 167

Urpin, V.A., Geppert U. \& Konenkov, D. Yu. 1998, MNRAS, 295, 907

Urpin, V.A. \& Konenkov, D. Yu. \& Geppert U. 1998, MNRAS, 299, 73

Wiringa, R.B., Fiks, V. \& Fabrocini, A. 1988, Phys.Rev.C, 38, 1010 